\title{
Human Neutrophils in Patients with Positive Serology for Chagas Disease
}

\section{Rodriguez $\mathrm{FM}^{1}$, Orquera $\mathrm{AD}^{1}$, Maturano $\mathrm{MR}^{1}$, Infante $\mathrm{NS}^{1}$, Carabajal-Miotti $\mathrm{C}^{2}$, Gonzalez-Silva $\mathrm{N}^{2}$ and Novak ITC ${ }^{* 1}$}

${ }^{1}$ Institute of Cell Biology, Faculty of Medical Sciences, National University of Cordoba, Argentina

${ }^{2}$ Institute of Hematology and Hemotherapy, National University of Cordoba, Argentina

*Corresponding author: Novak ITC, Enrique Barros corner Gordillo. Ciudad Universitaria, Cordoba (5000), Argentina, Tel: 0054351433 4020,E-mail: inovak@cmefcm.uncor.edu

Citation: Rodriguez FM, Orquera AD, Maturano MR, Infante NS, Carabajal-Miotti C, et al. (2016) Human Neutrophils in Patients with Positive Serology for Chagas Disease. J Immunol Infect Dis 3(1): 101

\section{Received Date: December 16, 2015 Accepted Date: April 26, 2016 Published Date: April 27, 2016}

\begin{abstract}
Polymorphonuclear (PMN) neutrophil leukocytes are cells of innate immunity recognized in a peripheral blood smear because of their particular multilobed nucleus. Ring shaped nucleous are typical in rats and mice, being described in some pathologies in humans as well as in healthy subjects. These neutrophils have been found early in Chagas disease patients. Here this study was conducted to observe the behavior and the morphology characteristics of PMN of autologous culture and on the other hand, its sex distribution in healthy individuals $(\mathrm{H})$ and with Chagas positive serology $(\mathrm{CH})$.

Materials and Methods: Quantification of ring cells in smears of heparinized human blood samples with and without positive serology for Chagas; testing with cytochemical reaction for myeloperoxidase (MPO) technique; autologous cell cultures; LPS assay to generating NETs, DNA visualized with DAPI in Immunofluorescence Microscopy; ultrastructural study with Transmission Electron Microscopy (TEM).

Results: No significant differences in blood smears in the number of neutrophils with ring nuclei between sexes were found in both groups $(\mathrm{H}$ and $\mathrm{CH})$. Significant differences $(p<0.0001)$ between the number of neutrophils with ring nuclei in $\mathrm{H}$ and $\mathrm{CH}$ were found. Neutrophils with ring nucleous were positive to myeloperoxidase in both groups. Ring cells were observed in 2 hours cultures. In $\mathrm{H}$ at $3 \mathrm{~h}$ of culture images of apoptosis were observed and at $20 \mathrm{~h}$ most of the PMN showed apoptotic characteristics. In $\mathrm{CH}$ after 72 hours of autologous culture viable PMN persisted. It could be due to persistence in the culture medium of cytokines and inflammatory mediators released by macrophages and lymphocytes further in samples from patients with positive serology for Chagas disease that prolong the half-life of PMN. We observed NETs in PMN with ring nuclei. We estimate that it could be highly interesting to pursue studies to determine the phenotypic profile of such neutrophils.
\end{abstract}

Keywords: Human neutrophils; Chagas disease; Ring shaped nuclei; Apoptosis; NETosis

\section{Introduction}

Recently it was reported that neutrophils participate in a key role in the effector and regulatory mechanisms of innate and adaptive responses [1-3]. Neutrophils are also called polymorphonuclear (PMN) because their nucleus may have three to five lobes connected by thin chromatin bridges. It has been proposed that the protein composition of the nuclear envelope of neutrophils allows them "deformability" of its nucleus and would facilitate the migration of activated cells from the vessels to sites of infection and also lobulation and forms in ring $[4,5]$. The existence of murine PMN with ring-shaped nucleus has been known for a long time, but in human neutrophils ring nucleus were observed earlier by Cabral in 1987 in chagasic patients and healthy individuals [6,7]. The ring shaped nuclei in neutrophils were composed of three or four areas lobed completely connected to each other, some of them by a thin strip of DNA. It was proposed that the large number (about 15\%) of these cells in peripheral blood suggested that they may be a normal evolutionary stage in the development of human PMN [6,7]. Moreover, in some pathologies, such as infectious mononucleosis, myelodysplastic syndromes and others, ring shaped nuclei were also observed in human neutrophils [8-15].

PMNs recognize microorganisms through various receptors and recognition promotes phagocytosis and then cytotoxic destruction [16]. The granules have a very diverse rich content and a set of degradative microbiostatic or microbicidal peptides such as myeloperoxidase (MPO) in the azurophilic granules [17]. In inflammatory conditions, PMNs trap and kill microbes through extracellular traps (NETs) composed of chromatin, histone and granule proteins [18-21]. NETs have been proposed as bridges to join the innate and adaptive immune responses by reducing the threshold of $\mathrm{T}$ cell activation [22]. It has also described the generation of NETs with sterile inflammatory stimuli from monosodium urate crystals [23]. NETs have also been implicated in thrombosis, tissue injury and autoimmunity [24-26]. The release of NETs can be "lytic NETosis suicidal" when it drifts PMN death or "vital NETosis" in the case of PMNs which are still alive [25,26]. It is not found in the literature report formation of NETs in neutrophils with ring shaped nuclei. 
Has been described neutrophils which release chemokines that attract T cells to sites of inflammation and [27-29] cytokines that influence the differentiation and proliferation increasing it in some cases and suppressing it in others [30,31]. IL17A and IL17F cytokines secreted by Th 17 cells induce rapid and massive infiltration of tissue affected by neutrophils [16]. In the case of parasitic infections such as Chagas disease, the role of family cytokines IL 17 is an area of current research [32].

Chagas disease is caused by the flagellate protozoan Trypanosoma cruzi and it is transmitted to humans by a blood-sucking bug Reduviidae family [33]. Infection with T. cruzi affects 10 million people in South and Central America and chronic cardiomyopathy is the main consequence of the infection, it developed in $30 \%$ of cases and immunological mechanisms are involved largely by the Th1 lymphocyte profile [34]. Some authors reported the involvement of 20 million people with Chagas disease in endemic areas of America and in recent years in North America and Europe immigration cases have been reported [32]. During the chronic stage of Chagas cardiomyopathy arrhythmias and conduction disturbances occur and can reach congestive heart failure; hypertrophy and aneurysms [35]. The neomicrovasculatura has been described as an active factor in the immunopathology of chronic Chagas disease [35]. Numerous studies have been published in relation to the pathogenesis of Chagas cardiomyopathy [35-37]. Bonney and Engman wonder if there are one or several pathogenetic mechanisms such as specific immunity induced by the parasite myocytolysis, primary neuronal damage, heart microvasculature damage, antibody-mediated cytotoxicity, no specific damage caused by eosinophils and neutrophils, among others [37]. The inflamatory cell infiltration in chagasic cardiac pathology consists mainly of lymphocytes, but macrophages, plasma cells, eosinophils and neutrophils were found too [37]. Although the persistence of the parasite in the myocardium and autoimmunity are primarily involved in the pathogeny, there is evidence of factors acting in synergy summatively and in disease development [37]. The hypothesis holds that heart damage initially leads to the breakdown of self-tolerance [32]. Regarding the neutrophil role in Chagas disease, it was experimentally demonstrated that murine PMNs stimulated in vitro with T. cruzi secreted IL-10 and showed a suppressive phenotype inhibiting T cell proliferation and IFN gamma secretion [32]. The increase of myeloperoxidase (MPO) activity and protein nitration as indicators of inflammation in patients with Chagas disease was described [38]. However, Voltarelli et al. have described decreased function of chemotactic activity and decreased oxidative capacity in neutrophils from patients with chronic Chagas [39]. The description of neutrophils with ring nuclei in greater numbers in Chagas disease [6] is striking and gives us the following questions: since they have been described in healthy people, what advantages can this leukocyte nuclear morphology bring in these particular circumstances? Regarding the nuclear morphology, an interesting study on the appearance of pathological nuclear forms in response to exposure to ionizing radiation in humans has been described recently [40]. On the role of neutrophils in Chagas disease there are relatively few studies, so it is very interesting data on the contribution of these cells. As regards longevity, PMNs in healthy subjects were considered short-lived cells [21]. However, at different times of autologous culture in previous studies PMN and plasma cells were observed at 48 hours in samples with positive serology Chagas disease [41]. Little information can be found about the involvement of neutrophils in T. cruzi infection. In murine models, it was described that neutrophils increase or reduce parasite burden in T. cruzi-infected macrophages, depending on host strain (strains of suceptible mices and strains of resistant mices). In infection resistense the action of neutrophil elastase, oxide nitric and TNFa by inducing microbicidal activity in infected macrophages are involved. Interaction with neutrophils regulates replication of T. cruzi parasite in macrophages from resistant and susceptible mice [42].

Here this study was conducted to observe the behavior and the morphology characteristics of PMN of autologous culture and on the other hand, its sex distribution in healthy individuals $(\mathrm{H})$ and with Chagas positive serology $(\mathrm{CH})$.

\section{Materials and Methods}

\section{Blood samples}

Heparinized human blood samples were collected with ethical consent according to procedures approved by ethical comitee of National Hospital Clinicas R: 107/12, Minute Book I, No 110, 12/04/12. Samples donated by the Blood Bank, Institute of Hematology and Hemotherapy of the National University of Cordoba in anonymity, with negative serology: Hudleson (Wiener), VDRL (Wiener), Chagas HAI (Wiener) Chagas EIE (Biomerieux), HBs EIE (Biomerieux), HBc (Biomerieux), HCV EIE (Murex), HIV Ac EIE (Biomerieux), HIV Ag EIE (Biomerieux), HTLV EIE (Murex). Individuals considered "healthy" (H) n = 30, 22 males and 8 females, age range 20-63; and only those with positive serology for Chagas $(\mathrm{CH}) \mathrm{n}=10 \mathrm{HAI}(+)$ and EIE (+), 6 males and 4 females, age range: $37-50$.

\section{Quantification of neutrophils with ring shaped nuclei (ring cells)}

We performed extended or whole blood smears, in all cases, and in samples of autologous cultures. The smears were fixed in ethyl alcohol 96 $6^{\circ}, 15$ minutes staining: hematoxylin / eosin (H/E). The neutrophils with ring nucleus were visualized by optic microscopy and the percentage of ring cells in a given citopreparation was calculated counting 100 neutrophils. The results are expressed as mean \pm standard deviation.

\section{Statistical analysis}

The data was presented as the mean value \pm SD, Student $t$ test was applied for data analysis ${ }^{\star *} p<0.0001$. The difference between means was analyzed using Infostat software. 


\section{Cytochemical reaction for myeloperoxidase (MPO)}

Fresh blood smears were air dried for 5 minutes. Covered extended with the following solution: benzidine (0.07 g) in sodium nitroprusside more saturated aqueous solution $(0.25 \mathrm{~mL})$ over $96^{\circ}$ ethanol $(24.8 \mathrm{~mL})$. It was allowed to act for $5 \mathrm{minutes}$. Without overturning the above solution, distilled water $(20 \mathrm{vol})$ was added $0.1 \%$. Then it was mixed blowing through pipette to homogenise both solutions and allowed it to stand for 2 minutes. It was washed with distilled Drying.

\section{Autologous cell cultures}

Total leukocyte cell cultures from circulating blood, suspended in sterile conical glass flasks, $37^{\circ} \mathrm{C}$ in TC199 medium (SIGMA, St. Louis, MO) added with filtered serum from the same donor. A 24-well cell culture plate was prepared by putting a sterile $13 \mathrm{~mm}$ round glass cover slip into each well to perform NETs formation assay and then observations in immunofluorescence microscope. Control of cell viability was performed by classical exclusion test with the dye Trypan Blue $0.5 \%$. Samples were taken at different times: 1, 2, 3, 20, 24, 48 and 72 hours of culture. The samples were fixed in ethyl alcohol $96^{\circ}$ for 15 minutes and stained with H/E for observation under the optical microscope. Some samples were processed for Transmission Electron Microscopy (TEM).

\section{NETs formation}

Cultured cells in a 24-well cell culture plate were stimulated to NETs formation with LPS (Sigma-Aldrich) [43] $25 \mathrm{ng} / \mathrm{ml}$ at $37^{\circ} \mathrm{C}$ in a $\mathrm{CO}_{2}$ incubator. Samples: $30 \mathrm{~min}$. Glass cover slips with attached cells were carefully removed from culture plate. They were washed briefly in PBS (phosphate buffered saline), fixation was performed with $4 \%$ paraformaldehyde for 10 minutes and washed in three changes in PBS. DNA staining with DAPI (4,6'-diamidino-2- phenylindole, Sigma, St Louis, MO). Mounting medium 90\% glycerol in PBS. Observation of preparations in immunofluorescence microscope, videomicroscope Axioscop 20, MC80, trinocular, Carl Zeiss. Paired blood samples provided controls.

\section{Transmission Electron Microscopy (TEM)}

Cell pellets were prepared with samples with aliquots of crops grown. They were fixed in $1 \%$ glutaraldehyde in $0.1 \mathrm{M}$ cacodylate buffer for one hour and post fixed in $1 \% \mathrm{OsO}_{4}$ in the same buffer for one hour. Then the materials were dehydrated in increasing graduation acetone and embedded in epoxy resin (Araldite) at $60{ }^{\circ} \mathrm{C}$ for $24-48$ hours. Later, ultrathin sections 60 were made to 80 $\mathrm{nm}$ thick (color silver / gold interference) that were collected on copper grids of 250 bars per inch, contrasted with uranyl acetate and lead citrate, and studied with electron microscope Zeiss LEO-906E transmission.

\section{Results}

\section{Quantification of ring cells}

The presence of neutrophils with ring shaped nucleus was positive both in circulating blood smears of $\mathrm{H}$ and $\mathrm{CH}$ (Figure 1). In all samples from $H$ the presence of ring cells was observed, with a range of 3 to 15\%, average: 7.90; SD: 2.77. Among females range of ring cells from 3 to 15\% was found, average: 8.87; SD: 3.52 and in males a range of ring cells of 5-13\%, mean: 7.54; SD: 2.44. The Student $\mathrm{t}$ test for small samples was performed and no significant difference of ring cells between healthy women and men was found. In all samples of $\mathrm{CH}$ the presence of neutrophils with ring nucleus was observed, with a range of 10-18\%, mean: 14.30; SD: 2.00. Among females, ring cells ranging from 10 to $15 \%$ was found, mean: 13.25 ; SD: 2.21 . In males a range of ring cells of $13-18 \%$, mean: 15; SD: 1.67. No significant differences of ring cells between sexes in patients CH were found. Significant differences of ring cells were found in the comparison of healthy people and positive serology for Chagas $\left({ }^{* *} p<0.0001\right)$ (Figure 1$)$.

\section{Results seen with myeloperoxidase (MPO) technique in ring cells}

It is noteworthy that the MPO positive granules of neutrophils are conspicuous and abundant in quantity, covering almost all the cytoplasm in brownish black color. The presence of this enzyme in ring cells of $\mathrm{H}$, as well as those of donors $\mathrm{CH}$ is highly positive comparable to that observed in polilobulated neutrophils in both groups (Figure 2).

\section{Ring cells in culture}

In samples at 2 hours of autologous leukocyte cultures the presence of neutrophils with ring shaped nucleus in $\mathrm{H}$ and $\mathrm{CH}$ was observed (Figure 2).

\section{Generating NETs in ring cells}

Cultured cells were stimulated to NETs formation with LPS and were observed in immunofluorescence microscope. In all tests of stimulation with LPS at 30 minutes generated NETs visualized as chromatin distribution in numerous fibrils or blue diffuse staining of DAPI (DNA) (Figure 2). We observed NET formation in ring cells in culture of patient CH without LPS stimulation (Figure 2). 


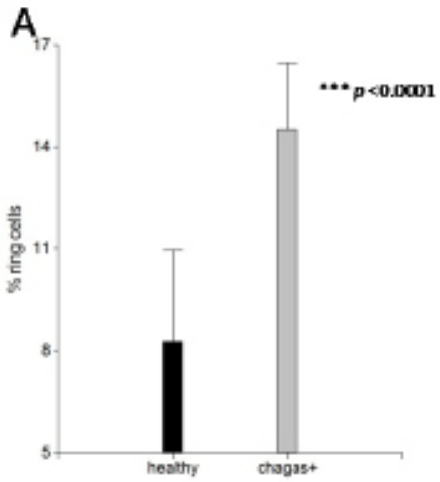

B

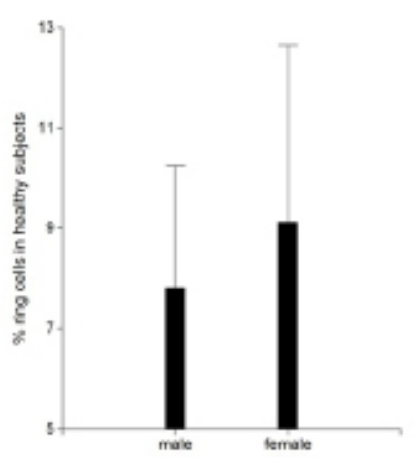

C

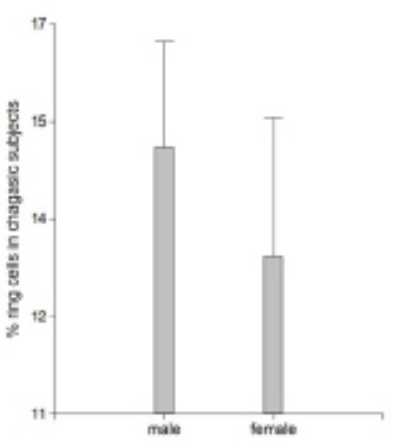

\begin{tabular}{|c|c|c|c|c|}
\hline & \multicolumn{2}{|c|}{$\begin{array}{c}\text { Ring cells in Healthy subjects } \\
\text { mean: } 7.90 \pm \text { SD: } 2.77 \\
n=30\end{array}$} & \multicolumn{2}{|c|}{$\begin{array}{c}\text { Ring cells in Chagasic subjects } \\
\text { mean: } 14.30 \pm \text { SD: } 2.00 \\
n=10 \\
\ldots .00,0001 \\
\end{array}$} \\
\hline & female & \begin{tabular}{|l|} 
male \\
\end{tabular} & female & male \\
\hline mean & 8.87 & 7.54 & 13,25 & 15.00 \\
\hline $\begin{array}{l}\text { SD (standard } \\
\text { deviation) }\end{array}$ & 3.52 & 2.44 & 2.21 & 1.67 \\
\hline
\end{tabular}

Figure 1: Quantification of ring cells. Neutrophils with ring nucleus were positive both in circulating blood smears of healthy people and people with positive serology for Chagas; (A) Significant differences were found in the comparison of healthy and positive serology for Chagas $(* * * p<0.0001)$; (B, C) No significant differences between sexes in both groups were found; (D) Descriptive statistic; The percentage of ring cells in a given citopreparation was calculated counting 100 neutrophils. The results are expressed as mean \pm standard deviation. Student $t$ test was applied for data analysis. ${ }^{* \star *} p<0.0001$
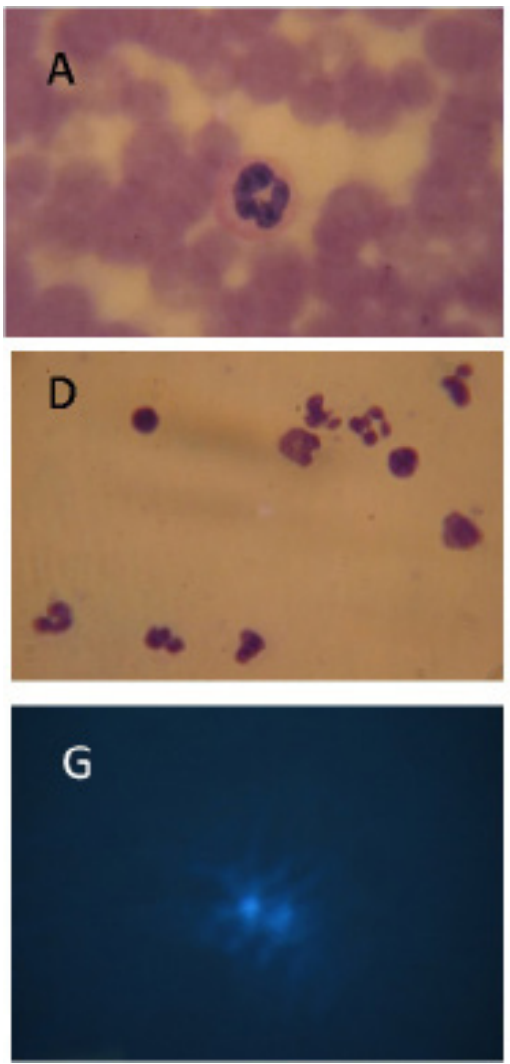
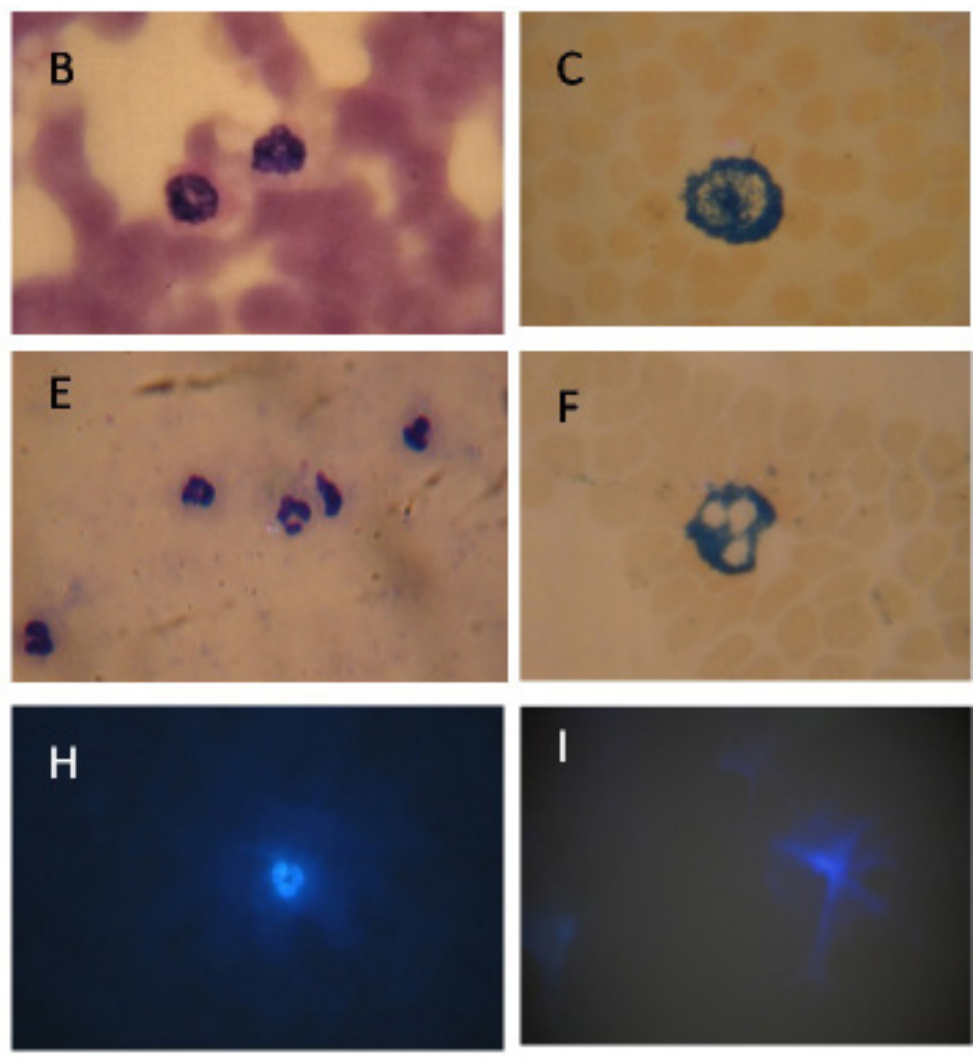

Figure 2: Images obtained with optical microscopy and immunofluorescence microscopy; (A) Neutrophil with ring nucleus in healthy subject 1000x; (B) Neutrophils with ring nucleus in subject with positive serology for Chagas 1000x; (C) Cytochemical reaction for myeloperoxidase (MPO) in neutrophil with ring nucleus in healthy subject 1000x; (D) Autologous leukocyte culture in healthy subject. Culture time: $1 \mathrm{~h}$ 600x; (E) Autologous leukocyte culture in subject with positive serology for Chagas. Culture time: $1 \mathrm{~h} \mathrm{600x;} \mathrm{(F)} \mathrm{Cytochemical} \mathrm{reaction} \mathrm{for} \mathrm{MPO} \mathrm{in} \mathrm{polilobulated} \mathrm{neutrophil} \mathrm{in} \mathrm{healthy} \mathrm{subject}$ 1000x; (G) ETs formation in autologous culture of blood leukocytes, in subject with positive serology for Chagas. LPS stimulation, 30 min. DNA (blue) DAPI (Sigma-Aldrich) 1000x; (H) ET formation in ring cell, without LPS stimulation, in autologous culture of blood leukocytes, in subject with positive serology for Chagas. DNA (blue) DAPI (Sigma-Aldrich) 1000x; (I) ET formation in autologous culture of blood leukocytes, in healthy subject. LPS stimulation, 30 min. DNA (blue) DAPI (Sigma-Aldrich) 1000x 


\section{Ultrastructural study of PMN in autologous leukocyte cultures}

Apoptotic neutrophils show condensation of the nucleus, chromatin margination and sometimes apoptotic bodies (Figure 3).

In $\mathrm{H}$, after 3 hours of culture we observed some neutrophils with apoptotic characteristics, on the other hand, at 20 hours most of the neutrophils were in apoptosis (Figure 3).
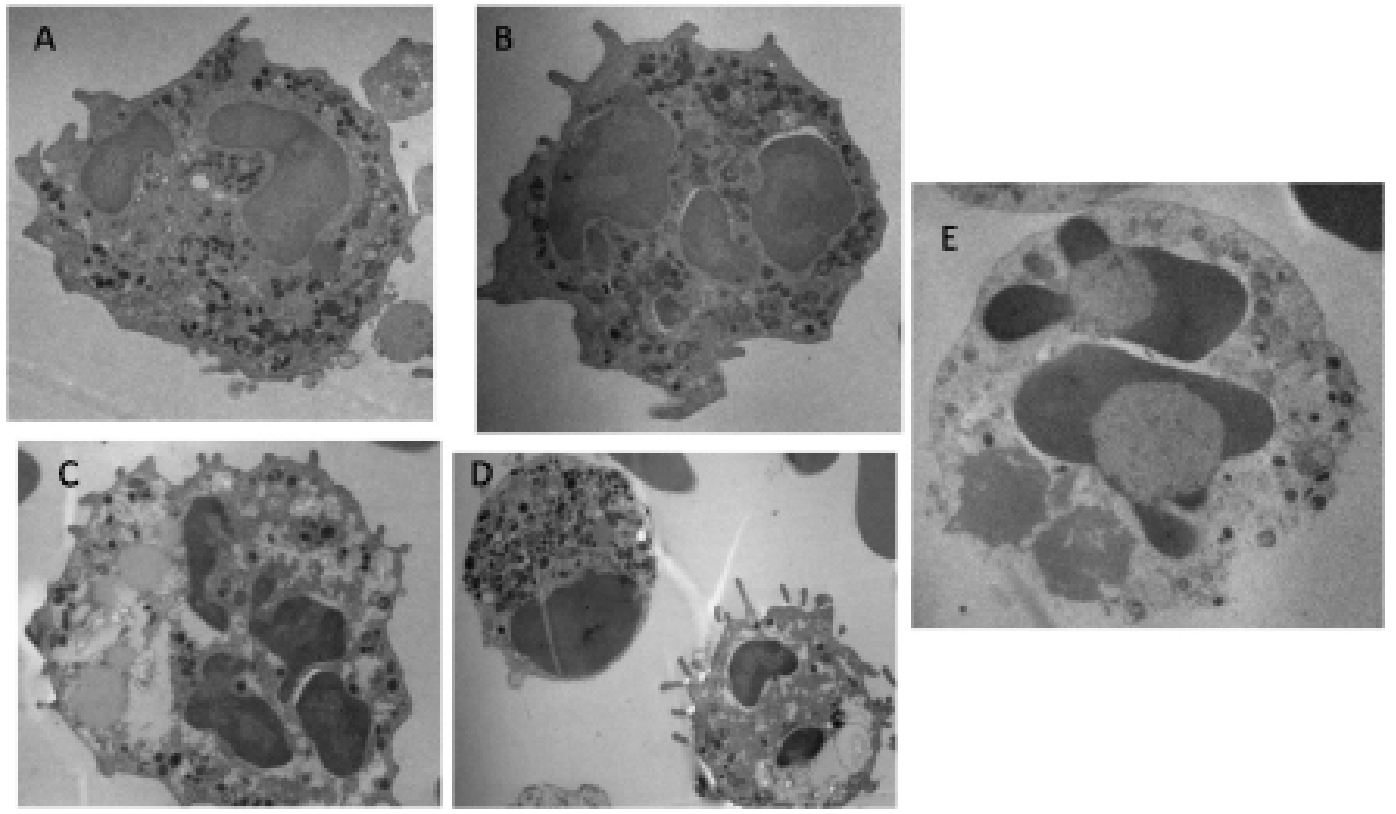

Figure 3: Representative transmission electronic microscopy (TEM) micrographs of neutrophils of healthy subject in autologous cell culture; (A, B) Culture time: 1 h 7750x; (C) Neutrophil with morphological changes. Culture time: 20 h 7750x; (D) Culture time: 24 h 6000x; (E) Neutrophil with characteristic morphology of apoptosis: chromatin margination, condensation of the nucleus. Culture time: $24 \mathrm{~h} 7000 \mathrm{x}$

In $\mathrm{CH}$, at 48 hours of autologous culture, we observed a NETosis image, the nuclear membranes were entirely fragmented while most of the granules were dissolved, allowing direct contact and mixing of nuclear, cytoplasmic, and granular components (Figure 4). After 72 hours of autologous culture, viable neutrophils with polilobulated nucleus persisted (Figure 4).
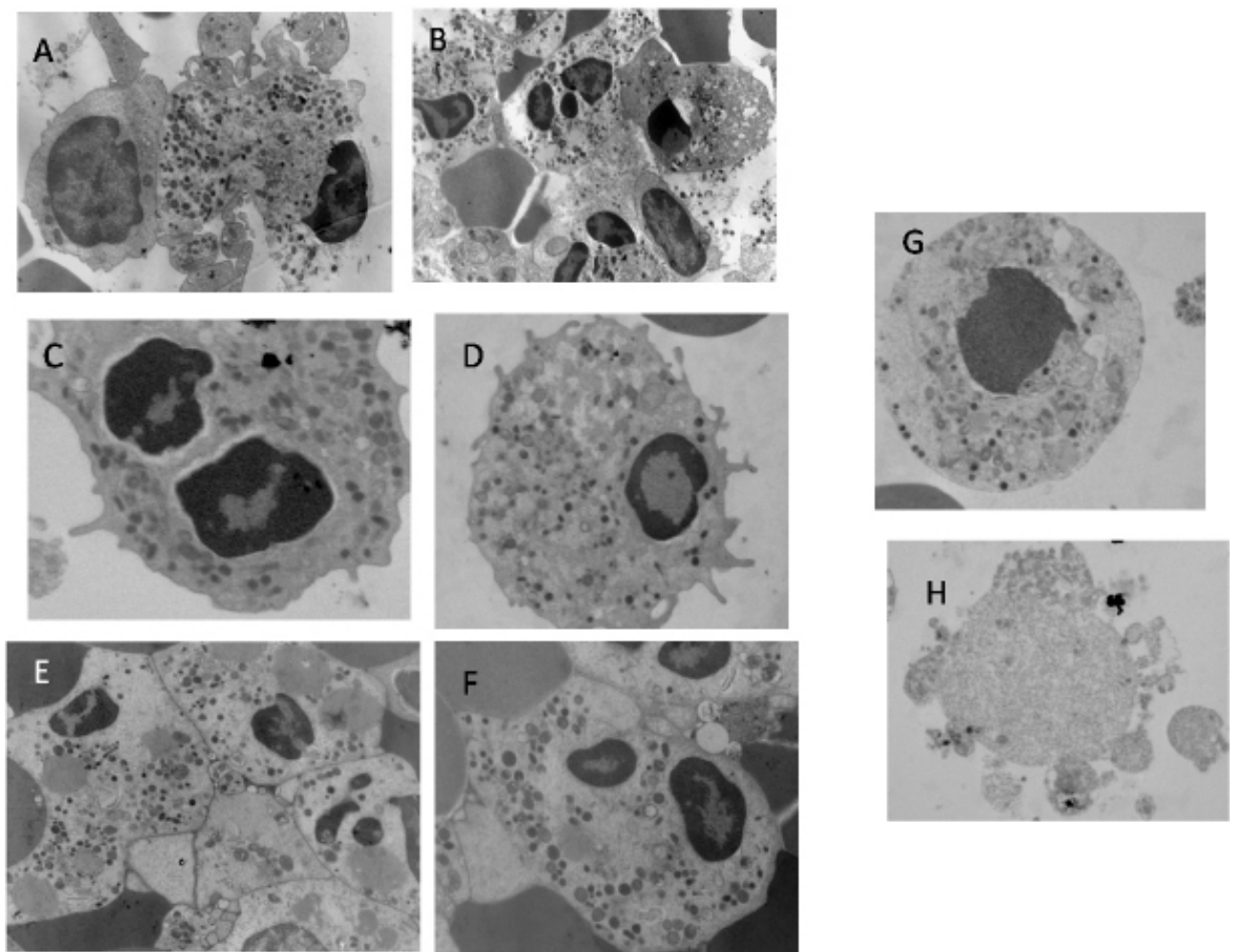

Figure 4: Representative transmission electronic microscopy (TEM) micrographs of neutrophils of subject with positive serology for Chagas in autologous cell culture; (A) Culture time: 24 h 7750x; (B) Culture time: 24 h 6000x; (C, D) Culture time: 48 h 7750x; (E) Culture time: 72 h 6000x; (F) Culture time: 72 h 7750x; (G) Neutrophil with characteristic morphology of apoptosis. Culture time: 48 h 7750x; (H) NETosis image. The nuclear membranes are entirely fragmented while most of the granules are dissolved, allowing direct contact and mixing of nuclear, cytoplasmic, and granular components. Culture time: $48 \mathrm{~h} 7750 \mathrm{x}$ 


\section{Discussion}

This study was conducted to observe the behavior and the morphology characteristics of PMN of autologous culture and on the other hand, its sex distribution in healthy individuals $(\mathrm{H})$ and with Chagas positive serology $(\mathrm{CH})$. Currently little is found in literature on neutrophils with ring shaped nucleus, as they are poorly described in some diseases and in healthy human few references allude [3-15]. In this study, we focused on finding neutrophils with ring shaped nucleus in peripheral blood of healthy humans and those with positive serology for Chagas; in order not only to observe their presence but to highlight aspects of their morphology and cytochemistry. The possibility of finding them in autologous cultures of total leukocytes from peripheral blood was also proposed.

We work with the Blood Bank of National University of Cordoba, Argentina, so most of the donors are healthy or asymptomatic people who donate their blood in a voluntary way. Only few cases of positive serology of Chagas occurred and they were informed by the Bank of Blood at that moment. We do not have treatment data afterwards. So we worked with a small sample with positive serology for Chagas. Most of these people came from the interior of the province of Córdoba. No significant differences in blood smears in the number of neutrophils with ring shaped nucleus between sexes were found in both groups. Significant differences $(p<0.0001)$ in the number of neutrophils with ring between $\mathrm{H}$ and $\mathrm{CH}$ were found in agreement with Cabral [6]. What is the functional significance of these nuclear forms? As stated, it has been proposed that it could be a normal evolutionary phase of normal neutrophil [7]. This shape favors diapedesis in response to secreted chemokines at sites of infection and inflammation [3-5]. These conditions may explain the highest number found in patients with positive serology for Chagas (Student's $t$ test, $p$ $<0.0001$ ). What might be the triggering stimuli for this "selection" of nuclear morphology Chagas disease? These questions deserve further study. Myeloperoxidase was positive in both groups $(\mathrm{H}$ and $\mathrm{CH})$. The presence of granulations is a feature that distinguishes neutrophils and granules are of great importance for its function. MPO housed in the azurophilic granules is part of the system of oxygen dependent cytotoxicity of these cells. The presence of this enzyme in ring cells of $\mathrm{H}$, as well as those of donors $\mathrm{CH}$ is highly positive comparable to that observed in polilobulated neutrophils in both groups. This high positivity could be related to a high increase in MPO activity, in agreement with another study in serum samples from chagasic patients [38]. The finding of MPO reactivity in neutrophil with ring shaped nucleus leads us to the microbicidal function thereof.

Here regarding viability, NETosis, or apoptosis at different times of autologous cultures, an ultrastructural study to observe the morphology characteristics of PMN was performed. PMNs in healthy subjects were considered short-lived cells [21]. With respect to PMNs with ring shaped nuclei, these cells were observed in healthy patiens in autologous total leukocyte cultures from peripheral blood at $2 \mathrm{~h}$. In this group we observed images of apoptosis from 3 hours of culture, at 20 hours, most of the PMNs with polilobulated nucleus showed apoptotic characteristics, but according to results of previous experiments in this laboratory they live 22 hours. After 72 hours of autologous culture, viable neutrophils with polilobulated nucleus persisted in patients with positive serology for Chagas. What stimuli contribute to increasing the average life of PMNs in Chagas? This significant difference could be due to the persistence in the culture medium (added with filtered serum from the same donor) of cytokines and inflammatory mediators released by macrophages and lymphocytes further in samples from $\mathrm{CH}$ patients to prolong the half-life of PMNs. We estimate of interest future studies to determine the phenotypic profile of such neutrophils.

We pose the question of whether the neutrophils with ring shaped nucleus will be able to form NETs as efficiently as polilobulated neutrophils do. It is well known that other leukocytes, besides the neutrophils, are able to generate extracellular traps (ET) [44]. In order to guarantee that these traps belong to neutrophils, it would be necessary to have a kind of marker, like neutrophil elastase. Here, taking into account the percentage of cells that emited traps, we can say that they would be neutrophils. In follow up studies marking will be done. We observed ETs formation in ring cells in culture of patient $\mathrm{CH}$ without LPS stimulation. We think that ETs generation would be related to presence of physiological inducters like antibodies or antibody-antigen complexes in the culture medium (added with filtered serum from the same donor) of $\mathrm{CH}$ group as inducers of ETs. We pose this question for further study: Is this ETosis "vital" or "suicidal"? We think it is very interesting to study the functional phenotype of ring cells, what type of cytokines do they secrete? Do they have an inflammatory or anti-inflammatory profile? Will they be IL-10 producers and will they behave as a suppressor phenotype?

This work allows supply of new data on human neutrophils and raises new questions regarding their origin and functional role in health and disease. We can say that there is a lot to discover about these interesting cells in the literature.

\section{Conclusion}

The study of the behavior and the morphology characteristics of PMN in healthy patients and with positive serology for Chagas allowed us to conclude: a) increased number of ring cells and high positivity to MPO in $\mathrm{CH}$; b) ETs formation in ring cells in culture of patients $\mathrm{CH}$ without LPS stimulation; c) increased longevity of polilobulated PMN in $\mathrm{CH}$. After 72 hours of autologous culture, viable neutrophils with polilobulated nucleus persisted in patients with positive serology for Chagas. What stimuli contribute to increasing the average life in culture of PMN in Chagas? That would be related to the presence of cytokines and inflammatory mediators in the serum of donors. We estimate that future studies to determine the phenotypic profile of such neutrophils will be extremely interesting. 


\section{Acknowledgements}

To the Blood Bank of Institute of Hematology and Hemotherapy, National University of Cordoba, Cordoba, Argentina for donating blood. We thank Professor Ana M. Granata for language editing of the work. We are also grateful to Reviewers whose comments helped us make amendments to our manuscript.

\section{References}

1. Perobelli SM, Galvani RG, Gonçalves-Silva T, Xavier CR, Nóbrega A, et al. (2015) Plasticity of neutrophils reveals modulatory capacity. Br J Med Biol Res 48: $665-75$.

2. Mantovani A, Cassatella MA, Costantini C, Jaillon S (2011) Neutrophils in the activation and regulation of innate and adaptive immunity. Nat Rev Immunol 11: 519-31.

3. Phillipson M, Kubes P (2011) The neutrophil in vascular inflammation. Nat Med 17: 1381-90.

4. Olins DE, Olins AL (2005) Granulocyte heterochromatin: defining the epigenome. BMC Cell Biol 6: 39

5. Hoffmann K, Sperling K, Olins AL, Olins DE (2007) The granulocyte nucleus and lamin B receptor: avoiding the ovoid. Chromosoma 116: 227-35.

6. Cabral HRA (1987) Neutrophils with ring-shaped nuclei in human Chagas' disease. Br J Haematol 67: 118-9.

7. Cabral HR, Robert GB (1989) Ring-shaped nuclei in human neutrophilic leukocytes of healthy individuals: evidence of their occurrence and characteristics. Am J Hematol 30: 259-60.

8. Kanoh, T (1991) Ring neutrophils in plasma cell dyscrasia. Arch Pathol Lab Med 115: 178-80.

9. Kanoh T, Saigo K, Yamagishi M (1986) Neutrophils with ring-shaped nuclei in chronic neutrophilic leukemia. Am J Clin Pathol 86: 748-51.

10. Schmitz LL, McClure JS, Litz CE, Dayton V, Weisdorf DJ, et al. (1994) Morphologic and quantitative changes in blood and marrow cells following growth factor therapy. Am J Clin Pathol 101: 67-75.

11. Peichev M (1986) Ring cells in infectious mononucleosis. Br J Haematol 62: 397-8.

12. Fenaux P (1996) Myelodysplastic syndromes. Hematol Cell Ther 38: 363-80.

13. Ruutu T (1986) Granulocyte function in myelodysplastic syndromes. Scand J Haematol 36: 66-70.

14. Kouides PA, Bennett JM (1996) Morphology and classification of the myelodysplastic syndromes and their pathologic variants. Sem Hematol 33: 95-110.

15. Lemež P, Kačírková P (2014) Variations of hairy cell nuclei shapes with regard to ring-shaped nuclei simulating dysplastic neutrophilic granulocytes and review of the literature. Int J Lab Hematol 36: 580-6.

16. Murphy KP (2012) Janeway's Immunobiology ( $8^{\text {th }}$ Edn) Garland Science, USA.

17. Borregaard N, Cowland JB (1997) Granules of the human neutrophilic polymorphonuclear leukocyte. Blood 89: 3503-21.

18. Brinkmann V, Reichard U, Goosmann C, Fauler B, Uhlemann Y, et al. (2004) Neutrophil extracellular traps kill bacteria. Science 303: 1532-5.

19. Brinkmann V, Zychlinsky A (2012) Neutrophil extracellular traps: is immunity the second function of chromatin? J Cell Biol 198: 773-83.

20. Branzk N, Papayannopoulos V (2013) Molecular mechanisms regulating NETosis in infection and disease. Semin Immunopathol 35: 513-30.

21. Kolaczkowska E, Kubes P (2013) Neutrophil recruitment and function in health and inflammation. Nat Rev Immunol 13: 159-75.

22. Tillack K, Breiden P, Martin R, Sospedra M (2012) T Lymphocyte priming by neutrophil extracellular traps links innate and adaptive immune responses. J Immunol 188: 3150-9.

23. Schorn C, Janko C, Krenn V, Zhao Y, Munoz LE, et al. (2012) Bonding the foe - NETting neutrophils immobilize the pro-inflammatory monosodium urate crystals. Front Immunol 3: 10.3389/fimmu.2012.00376. eCollection 2012.

24. Barrientos L, Marin-Esteban V, de Chaisemartin L, Le-Moal VL, Sandré C, et al. (2013) An improved strategy to recover large fragments of functional human neutrophil extracellular traps. Front Immunol 4: 10.3389/fimmu.2013.00166. eCollection 2013.

25. Kumar V, Sharma A (2010) Neutrophils: Cinderella of innate immune system. Int Immunopharmacol 10: 1325-34.

26. Yipp BG, Kubes P (2013) NETosis: how vital is it? Blood 122: 2784-94.

27. Scapini P, Lapinet-Vera JA, Gasperini S, Calzetti F, Bazzoni F, et al. (2000) The neutrophil as a cellular source of chemokines. Immunol Rev 177: 195-203.

28. Müller I, Munder M, Kropf P, Hänsch GM (2009) Polymorphonuclear neutrophils and T lymphocytes: strange bedfellows or brothers in arms? Trends Immunol 30: 522-30.

29. Pelletier M, Maggi L, Micheletti A, Lazzeri E, Tamassia N, et al. (2010) Evidence for a cross-talk between human neutrophils and Th17 cells. Blood 115: $335-43$. 30. Denkers EY, Del Rio L, Bennouna S (2003) Neutrophil production of IL-12 and other cytokines during microbial infection. Chem Immunol Allergy 83: 95-114.

31. Thewissen M, Damoiseaux J, van de Gaar J, Tervaert JW (2011) Neutrophils and T cells: bidirectional effects and functional interferences. Mol Immunol 48: 2094-101.

32. Boari JT, Vesely MCA, Bermejo DA, Ramello MC, Montes CL, et al. (2012) IL-17RA Signaling Reduces inflammation and mortality during Trypanosoma cruzi infection by recruiting suppressive IL-10-producing neutrophils. PLoS Pathog 8: e1002658.

33. Coura JR (2015) The main sceneries of Chagas disease transmission. The vectors, blood and oral transmissions--a comprehensive review. Mem Inst Oswaldo Cruz 110: 277-82.

34. Ferreira LR, Frade AF, Baron MA, Navarro IC, Kalil J, et al. (2014) Interferon- $\gamma$ and other inflammatory mediators in cardiomyocyte signaling during Chagas disease cardiomyopathy. World J Cardiol 6: 782-90.

35. Cabral HRA (1969) Mecanismos patogenéticos del daño tisular en Chagas [Pathogenic mechanisms of tissue damage in Chagas’s disease]. Rev Fac Cienc Méd Córdoba 27: 287-309.

36. Cabral HR, Novak IT, Glocker M, Castro-Viera GA (2005) Neomicrovasculatura: factor activo en la inmunopatogenia de la cardiopatía crónica chagásica [Neomicrovasculatura: active factor in the immunopathology of chronic Chagas disease]. Rev Argent Cardiol 73: 201-7. 
37. Bonney KM, Engman DM (2008) Chagas heart disease pathogenesis: one mechanism or many? Curr Mol Med 8: $510-8$.

38. Dhiman M, Estrada-Franco JG, Pando JM, Ramirez-Aguilar FJ, Spratt H, et al. (2009) Increased myeloperoxidase activity and protein nitration are indicators of inflammation in patients with Chagas' disease. Clin Vaccine Immunol 16: 660-6.

39. Voltarelli JC, Donadi EA, Carvalho IF, Falcão RP (1990) Lymphocyte subpopulations and neutrophil function in chronic human Chagas' disease. Rev Inst Med trop São Paulo 32: 240-8.

40. Goans RE, Iddins CJ, Christensen D, Wiley A, Dainiak N (2015) Appearance of pseudo-Pelger Huet anomaly after accidental exposure to ionizing radiation in vivo. Health Phys 108: 303-7.

41. Novak ITC, Orquera AD (2013) Ultrastructure of human autologous macrophage lymphocyte rosettes in Chagas disease. Front Immunol Conference Abstract: 15th International Congress of Immunology (ICI): 10.3389/conf.fimmu.2013.02.00875.

42. Luna-Gomes T, Filardy AA, Rocha JD, Decote-Ricardo D, LaRocque-de-Freitas IF, et al. (2014) Neutrophils increase or reduce parasite burden in Trypanosoma cruzi-infected macrophages, depending on host strain: role of neutrophil elastase. Plos One 9: e90582.

43. Lim MB, Kuiper JW, Katchky A, Goldberg H, Glogauer M (2011) Rac2 is required for the formation of neutrophil extracellular traps. J Leukoc Biol 90: 771-6.

44. Muñoz-Caro T, Rubio R MC, Silva LM, Magdowski G, Gärtner U, et al. (2015) Leucocyte-derived extracellular trap formation significantly contributes to Haemonchus contortus larval entrapment. Parasit Vectors 8: 10.1186/s13071-015-1219-1.

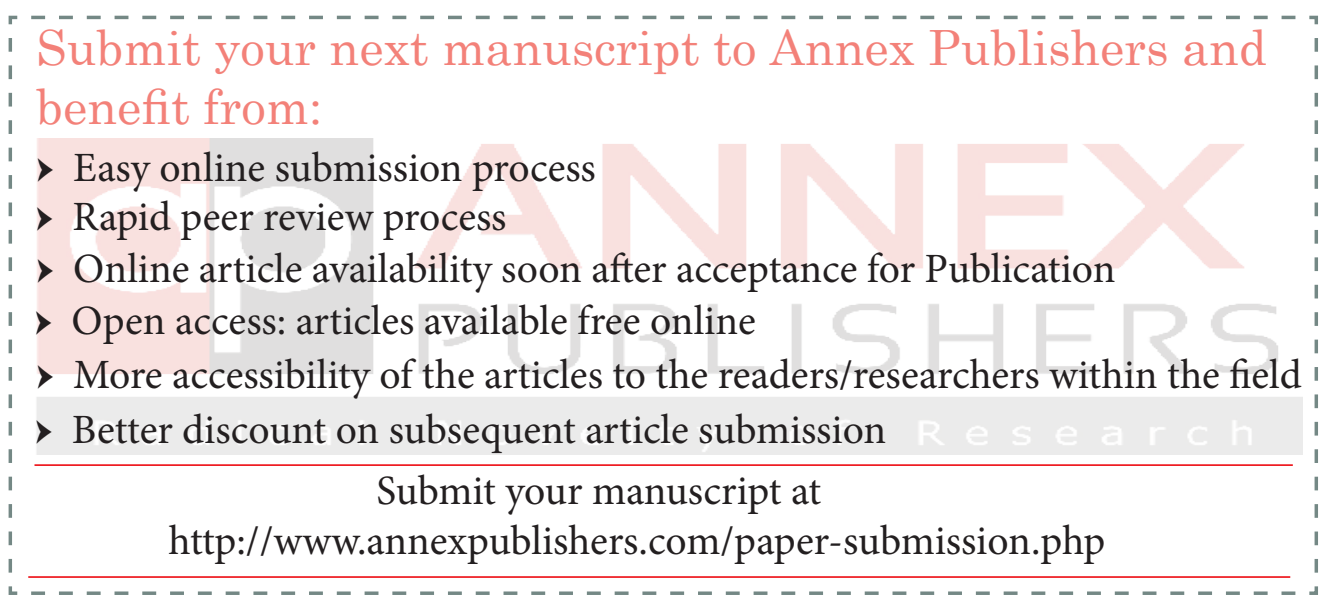

